\title{
Coordinate diurnal variation in the strength of startle elicitation and of startle modification in the rat
}

\author{
JAMES R. ISON \\ University of Rochester, Rochester, New York \\ and \\ JOHN A. FOSS \\ Bristol-Myers Squibb Pharmaceutical Research Institute, New Brunswick, New Jersey
}

\begin{abstract}
In order to determine the effect of time of day on reflex modification, we measured the acoustic startle reflex (ASR) in rats $(n=4)$ to 120 - $\mathrm{dB}$ tone bursts given alone to provide the ASR baseline, $100 \mathrm{msec}$ after an inhibiting brief noise prepulse, or 10 to $20 \mathrm{sec}$ after the onset of a facilitating noise background for 4-5 days in a test environment illuminated on a 12:12-h light:dark cycle. ASR amplitudes were greater in the dark, and sinusoids with a 24-h period starting at the light:dark transition accounted for about $80 \%$ of the variance in ASR amplitudes within each stimulus condition. The absolute differences in amplitude between baseline and either inhibited or facilitated ASR means were also greater in the dark, but relative measures of reflex modification (expressed as a proportion of the ASR baseline) were constant across time of day save for a slight compressive nonlinearity. The outcome for difference scores indicated that those neural mechanisms responsible for ASR elicitation and those responsible for ASR modification by external stimuli are both controlled in part by time of day, and the outcome for the relative response scores indicated further that the sensitivity of these separate reflex control mechanisms to the circadian process must be about equal.
\end{abstract}

The strength of the acoustic startle reflex (ASR) in the rat expresses a circadian rhythm, reaching its peak during the night and its trough during the illuminated daytime hours (Chabot \& Taylor, 1992a, 1992b; Davis \& Sollberger, 1971; Horlington, 1970). The ASR is also sensitive to shifts in the external stimulus surround, being inhibited by brief stimuli presented just prior to reflex elicitation (see the review by Hoffman \& Ison, 1980), but facilitated by more prolonged stimuli, at least after their initial inhibitory effect has dissipated (Davis, 1974; Gerrard \& Ison, 1990; Hoffman, Marsh, \& Stein, 1969; Hoffman \& Searle, 1965; Hoffman \& Wible, 1970; Ison \& Hammond, 1971; Ison, McAdam, \& Hammond, 1973). Here we examined how the cyclic diurnal variation in response strength that is independent of the contemporary stimulus environment present in the testing cage may interact with those mechanisms that are sensitive to the shifting stimulus surround and are then responsible for the inhibitory and facilitatory modulation of the ASR. The question of interest is whether the mechanisms that produce reflex modulation are independent of those responsible for the circadian rhythm, or otherwise, wax and wane in concert with time

This research was supported by USPHS Research Grant AG09524 and a Center Support Grant to the Center for Visual Science at the University of Rochester, EY01319. Correspondence should be addressed to J. R. Ison, Meliora Hall, University of Rochester, Rochester, NY 14627 (e-mail: ison@bcs.rochester.edu).

-Accepted by previous editor, Paul E. Gold of day and the coordinate changes in ASR amplitudes. If reflex modification expresses the circadian rhythm that affects reflex elicitation, absolute differences between the baseline ASR and the inhibited and facilitated response amplitudes should peak during the night. Further, if reflex modification and reflex elicitation are equally sensitive to the same circadian rhythm, relative measures of prepulse inhibition (PPI) and prepulse facilitation (PPF) (in which reflex modification is quantified as a proportion of the baseline ASR) will remain constant throughout the day despite the expected systematic variation in ASR amplitude and despite systematic variation in difference score measures of reflex modification. Rusek (1981) described diurnal variation in a large number of behavioral and physiological measures, and it would be surprising if reflex modification were not to be numbered among this group: But this outcome is not certain, and the question has not been examined previously.

\section{METHOD}

\section{Subjects}

The subjects were 4 naive male Long-Evans rats, 5-7 months old. The animals were born and raised in the vivarium at the University of Rochester, on a 12:12-h light:dark cycle with ad-lib food and water. The lights were turned on at $0900 \mathrm{~h}$ each morning and off at $2100 \mathrm{~h}$ each evening.

\section{Apparatus}

The subject was confined for testing in a wire-mesh cage $(17.5 \mathrm{~cm}$ wide, $24 \mathrm{~cm}$ long, and $17.5 \mathrm{~cm}$ high) that was suspended above a 
floor of metal rods attached to a stiff acrylic shelf in a small soundattenuating chamber (inside dimensions: $35 \times 45 \times 60 \mathrm{~cm}$ ). An accelerometer (Model SA-2-300, Statham Laboratories, Beverly Hills, CA) attached to the bottom of the shelf was sensitive to the force exerted on the platform by the startle response. The output of the accelerometer was passed through a bridge amplifier and integrated over an 80 -msec period beginning with the startle stimulus. Two round holes in the cage $(2.5-\mathrm{cm}$ diameter) allowed the animal to have access to the drinking spout of a water bottle and to a bin of food pellets (45-mg pellets, P. J. Noyes Co., Inc., Lancaster, NH). A photocell placed across the food bin and a drinkometer circuit running through the water spout and the floor of the test chamber permitted the recording of drinking bouts and eating (which was defined by nosepokes into the food hopper). The chamber was illuminated by a remotely controlled $40-\mathrm{W}$ incandescent bulb placed just outside the translucent window of the small chamber, and was housed inside a single-walled sound-attenuating room $(1.9 \times 2.8 \times 2.5 \mathrm{~m})$. The acoustic startle stimulus was a $25-\mathrm{msec}$ ( $5 \mathrm{msec}$ rise/fall time), 120-dB (SPL), 10-kHz tone burst provided by a tweeter. The preliminary stimuli were both $65-\mathrm{dB}$ noise bursts provided by a white noise generator constructed at the University of Rochester, shaped by an electronic switch to have 5-msec rise and decay times, amplified, and then delivered through a mid-range speaker: These stimuli varied by no more than $\pm 2 \mathrm{~dB}$ from $1 \mathrm{kHz}$ to $32 \mathrm{kHz}$, measured across 1 -octave bands. All prepulse measurements were made with a Brüel \& Kjaer sound level meter (Model 2203) with an external octave filter and Model 4165 microphone, and the startle stimulus was measured with a General Radio Company sound level meter (Model 1561) with an impact noise analyzer (Model 1556-B) and Type 1560-P7 microphone. The calibration of the stimuli was checked at the beginning of the experiment (the equipment is on continuously and routine calibration checks have shown that these values are stable over many months unless the amplifier settings are changed). A computer was responsible for response recording and the stimulus control of the experiment.

\section{Procedure}

The experimental chamber was illuminated from 0900 to $2100 \mathrm{~h}$ each day. The subject was placed in the chamber variously from 12 to $24 \mathrm{~h}$ prior to testing. The experiment began at $2100 \mathrm{~h}$, and then continued for the next $4 \frac{1}{2}$ to $51 / 2$ days, with the subjects removed from the apparatus at different times during the last day. The subjects were not disturbed for this duration, save that the experimenter opened the smaller chamber briefly at the end of each 12 -h segment in order to check the food and water supply. Startle stimuli were given at a rate of 12 per hour over the course of the experiment, for 2 subjects $5 \mathrm{~min}$ apart, to run evenly through the hour, and for 2 subjects 1-2 min apart, with a run completed within about $20 \mathrm{~min}$. Brief delays were inserted into these schedules by a requirement that startle stimuli were never presented within $2 \mathrm{sec}$ of drinking or an entrance to the food bin. Three stimulus conditions were used. One condition provided the baseline control and consisted of the startle stimulus presented alone. The second condition was intended to provide a measure of startle facilitation and consisted of the startle stimulus presented in the presence of a prolonged background noise that lasted for $10-20 \mathrm{sec}(M=15 \mathrm{sec})$ and ended $200 \mathrm{msec}$ after the startle stimulus. The third condition was intended to provide a measure of reflex inhibition and consisted of the startle stimulus being presented $100 \mathrm{msec}$ after the onset of a brief noise prepulse that lasted for $20 \mathrm{msec}$. The three conditions were presented in semirandomized blocks so that none was repeated until each had been presented on an equal number of trials.

\section{Data Analysis}

The data reported here are taken from the last 3 test days of each animal, with the presumption that response levels would be relatively stable after the subjects were adapted to the startle stimuli and the test environment. Responses for each condition were gath- ered into 2-h blocks over the 3 days and then summed for each time block over days to provide 36 scores, one for each of the twelve 2-h periods for each of the three stimulus conditions. The scores were then rearranged for analysis to begin at the onset of the dark period. The resulting mean response amplitudes were then normalized (by expressing each subject's mean in each condition as a proportion of that subject's summed responses over all conditions) in order to eliminate individual differences in overall response amplitude, while maintaining the proportional differences between conditions. Because the subjects were removed from the apparatus at different times, the dark periods came on average earlier in the experimental trials for 2 subjects and later for the others, thus avoiding the otherwise necessary confounding of habituation and time of day (as all testing began at $2100 \mathrm{~h}$ ).

These data were subjected to a within-subjects analysis of variance (ANOVA), in which light versus dark (LD), hours (H, within light vs. dark), and stimulus conditions (SC) were the three dimensions of interest. (The Huynh-Feldt adjustment of degrees of freedom was used to correct for any differences in the correlation coefficients across cells in the ANOVA.) A significant effect for LD without an accompanying effect of $H$ would suggest that the ASR varied according to the simple presence or absence of the light, whereas a significant $\mathrm{LD} \times \mathrm{H}$ interaction would reveal the presence of a circadian rhythm in which ASR amplitudes changed in anticipation of the next lighting period. Significant interactions of SC with $\mathrm{LD}$ or $\mathrm{LD} \times \mathrm{H}$ would reveal that the absolute differences between the three stimulus conditions varied with the diurnal cycle. A second transformation of the data calculated the difference scores for the means between each of the prestimulus conditions and the control condition, and a third divided the mean scores for the prepulse trials for each subject for each time period by that subject's mean score for the baseline condition, to provide measures of relative responding. (These are the two common transformations of response amplitudes that are used to quantify reflex modification.) The difference scores were used for graphic purposes but were not separately analyzed since the main effects in their ANOVA are quantitatively identical to certain interactions in the ANOVA of the ASR amplitudes. The relative scores were used for graphic purposes and also were subjected to the ANOVA. In this ANOVA a significant effect of LD and $\mathrm{H}$ would reveal that relative measures of reflex modification varied according to a diurnal rhythm and thus would indicate that the effects of the diurnal rhythm on reflex modification and reflex elicitation were not identical. A set of correlational analyses was additionally provided to follow up interesting patterns of effects for $\mathrm{LD}$ and $\mathrm{H}$ found in the ANOVA. For these analyses we calculated the best-fitting sinusoid to the baseline control data and then determined the amount of variance accounted for in ASR amplitudes by this sinusoid, or determined the amount of variance accounted for in the relative scores by the obtained control ASR amplitudes.

\section{RESULTS}

Figure 1 provides the normalized mean response amplitudes $( \pm 1 S E M)$ for each of the three stimulus conditions, presented in 2-h blocks summed over the last 3 test days. In addition, a "best-fit" $24-\mathrm{h}$ period sinusoid based on the control data is presented for each function, with its amplitude, but not its phase or period, adjusted for that particular function. In accord with the prior findings, both prepulse conditions and the light:dark cycle affected ASR amplitude: The brief pulse inhibited and the prolonged noise facilitated the ASR, and the ASR was generally greater in the dark than in the light. The ASR appeared to follow an internal rhythm rather than the simple presence absence of light, because it reached its trough in the early 


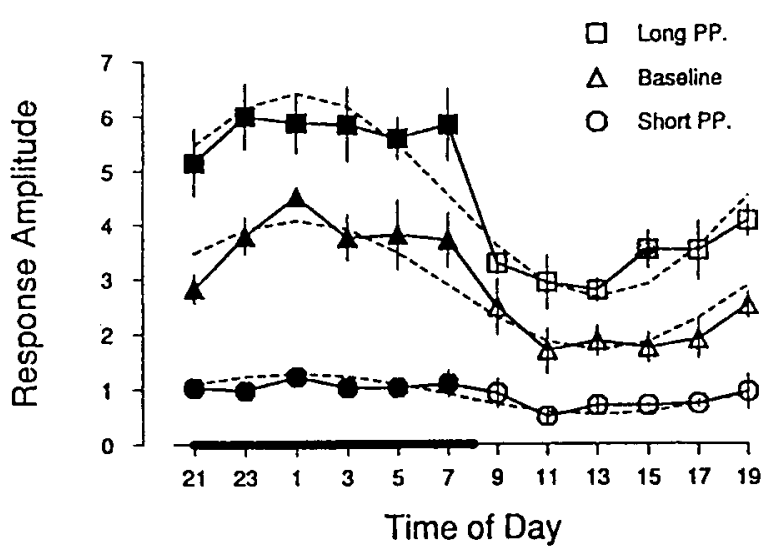

Figure 1. Normalized mean startle response amplitudes $( \pm S E M)$ for each 2-h period within the day, summed over 3 test days, for each of three conditions: a facilitatory condition in which a 10- to 20-sec noise burst overlapped the startle burst (square symbols); a baseline control condition, with the startle stimulus alone (triangles); and an inhibitory condition, in which a 20 -msec noise burst was present 100 msec before the startle stimulus (circles). The sinusoids approximate to each function have the period and phase of the baseline control data, with their amplitudes adjusted to match each function. Filled symbols denote the dark period.

part of the light period and then began its rise toward the dark period peak prior to light offset. Similarly, the response increased to its peak during the early part of the dark period, but the subsequent decline toward the trough in anticipation of light onset was less marked than was the anticipation of dark onset. Overall the sinusoidal function described by the control data agrees quite well with that presented by Sollberger and Davis (1972), with its extreme values occurring just short of midway through each phase of the cycle, even though their data were based on startle response probabilities (defined against an arbitrary criterion) rather than startle amplitudes. Figure 2 presents the transformed values of these data, the difference scores $(R c-R p)$ in the top panel and the relative scores $(R p / R c)$ in the lower panel. In the upper panel, the horizontal lines give the overall mean differences between the control value and the response obtained in the presence of the background noise $(+1.65 \pm .24)$ and following $100 \mathrm{msec}$ after the brief noise burst $(-1.98 \pm .41)$, and the boxes on these lines depict $\pm 1 S E M$, calculated on the null hypothesis that the mean values were not different from the overall mean. Absolute differences between baseline and inhibited ASR values were substantially greater in the dark than in the light, whereas the same trend for facilitation produced by the long prepulse condition was less pronounced. In the bottom panel of Figure 2, one horizontal dotted line depicts the mean overall facilitated response as a relative figure (at $173.2 \%$ of the control value, i.e., $73.2 \%$ facilitation) and the other depicts the mean inhibited response (at $34.2 \%$ of the control value, i.e., $65.8 \%$ inhibition). The centered sinusoidal curve has the period and phase of the ASR amplitudes and is presented to provide the reference for the effect of time of day on amplitude measures. It is apparent in these relative data that any effects of the circadian rhythm that are specific to reflex modification compared with reflex elicitation must be slight, as few of these relative response means departed from their associated horizontal line by more than $1 S E M$. In this respect, the relative measures of reflex modification are sharply differentiated from the absolute difference scores.

The ANOVA of the data presented in Figure 1 yielded significant main effects of SC $[F(2,6)=62.05, p<.01]$ and $\operatorname{LD}[F(1,3)=92.99, p<.01]$, which entered into a significant interaction $[\mathrm{SC} \times \mathrm{LD}, F(2,6)=21.64, p<.01]$. The main effect for $\mathrm{H}$ was not significant $(F<1)$, but this variable entered into a significant interaction with $L D$, for an $\mathrm{LD} \times \mathrm{H}$ (quadratic trend) interaction $[F(1,3)=52.35, p<$ $.01]$. This first interaction shows that reflex modification, for PPI, for PPF, or for both effects, varied with LD; and the second interaction shows that the ASR within the dark or light periods varied with $\mathrm{H}$, indicating that the circadian rhythm was independent of the current lighting condition.

To test for the factors responsible for the SC $\times$ LD interaction, a separate ANOVA analyzed the inhibitory data
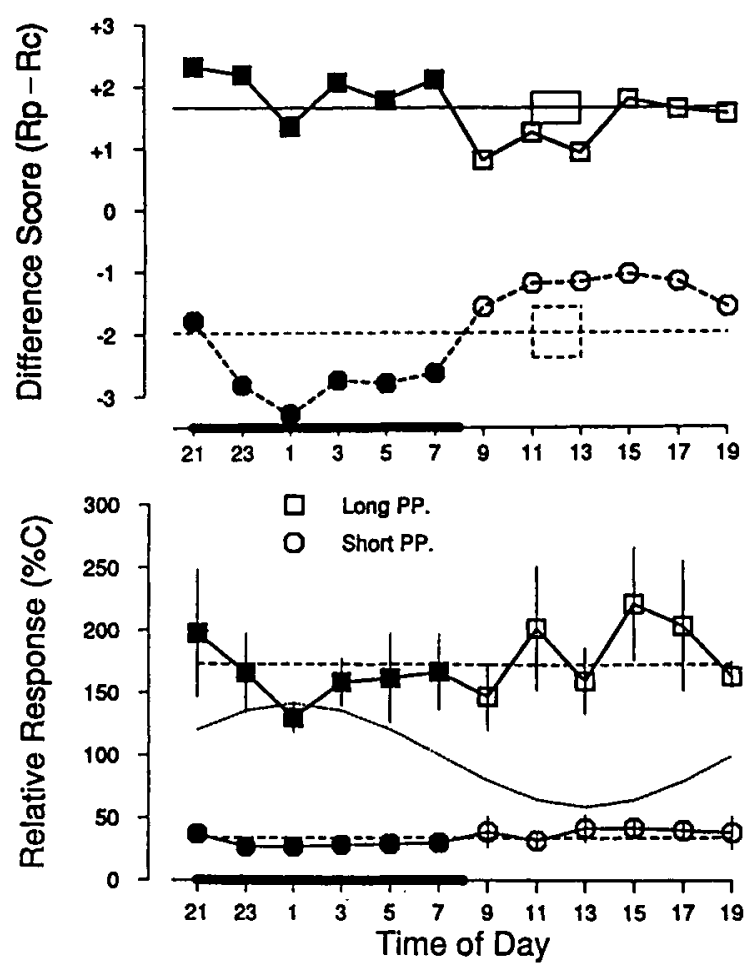

Figure 2. Top panel: difference scores obtained by subtracting the mean response obtained in the control condition $(\mathrm{Rc})$ from the response in each prepulse condition ( $R p)$. The horizontal dotted lines give the overall mean difference scores between conditions, and the boxes on the lines represent $\pm S E M$ given the null hypothesis. Bottom panel: relative response amplitudes $( \pm S E M)$ on facilitated and inhibited trials for each 2-h period within the day, with the responses for each subject in each prepulse condition expressed as a proportion of its control response in the same time period. Running through the center is the normalized sinusoid that accounted for $83 \%$ of the variance in the control baseline condition. 
for the brief prepulse against the control and the facilitatory data for the prolonged background against the control. For the brief prepulse there was a significant main effect for $\mathrm{SC}[F(1,3)=113.67, p<.01]$, confirming that reliable PPI was present in these data, and for $\operatorname{LD}[F(1,3)$ $=48.56, p<.01]$, confirming the facilitatory effect of dark compared with light for the ASR, and for the SC $\times$ LD interaction $[F(1,3)=17.84, p<.05]$, showing that the difference between baseline and inhibited ASR values, as presented in the upper panel of Figure 2, was greater in the dark than in the light. For the background noise, there was a significant effect for $\mathrm{SC}[F(1,3)=12.90, p<.05]$, attesting to the reliability of facilitation, and for $\operatorname{LD}[F(1,3)$ $=70.55, p<.01]$, showing again the positive effect of dark on the ASR; but the interaction was not significant $[F(1,3)=4.13, p>.1]$. The failure to show a significant interaction for PPF in this last ANOVA is problematical, however, because although each of the 4 subjects showed greater facilitation in the dark, this effect in 2 animals was very large and seemed to be responsible for the unusual variance obtained in this comparison. In order to reduce the degree to which the data were skewed by these extreme values, the difference scores for facilitation in light versus dark were subjected to a square root transformation, intended specifically to reduce the contribution of the extreme positive scores to both the variance and the mean. The analysis of the resulting transformed scores was significant $[t(3)=3.14, p=.05]$. A reasonable conclusion, then, is that reflex modification-that is, both reflex facilitation and reflex inhibition as depicted in the difference scores in Figure 2-is more pronounced in the dark than in the light.

The relative response data presented in the lower panel of Figure 2 were also subjected to an ANOVA in which the dimensions of interest were $\mathrm{SC}, \mathrm{LD}$, and $\mathrm{H}$. This analysis provided a significant effect only for SC $[F(1,3)=$ $30.98, p=.01$ ], whereas the main effects for $\mathrm{LD}$ and $\mathrm{H}$, the interaction $\mathrm{LD} \times \mathrm{H}$, and the several interactions of $\mathrm{SC}$ with these main effects and their interaction were not significant $(p>.1)$. Thus, the ANOVA provided no inferential support for the hypothesis that the circadian rhythm expressed in reflex modification is any different from that of reflex elicitation. However, the two relative response functions, although they do not deviate significantly from the horizontal, do suggest a small but potentially interesting patterned effect, which is that the relative values for the inhibited condition and the facilitated condition were both slightly smaller when the ASR was at its peak in the dark: 3 of the 4 subjects had less facilitation in the dark (with means of $63.4 \%$ in the dark vs. $83.0 \%$ in the light), whereas all 4 subjects showed greater inhibition in the dark ( $70.3 \%$ in the dark vs. $61.3 \%$ in the light). Neither of these differences is significant given any transformation of the data $(p>.1)$, but it is of some interest that the control ASR means for the 12 time points correlated negatively with the mean values for facilitation $(r=-.68, p<$ $.05)$ and positively with the mean values for inhibition $(r=+.85, p<.01)$. This many statistical analyses might be considered overly aggressive in the search for some inferential support for the small patterned effect shown in the relative scores of Figure 2, but they can be justified as an appropriate alternative to accepting the "nonsignificant" effect of diurnal rhythmicity of relative reflex modification found in the ANOVA as evidence for the null hypothesis. In fact, even taken at face value, they suggest not a general effect of time of day on reflex modification, but instead a small "compressive nonlinearity" in the data, indicating that large responses obtained in the dark are more difficult to facilitate but easier to inhibit, whereas the smaller responses obtained in the light are relatively easy to facilitate but more difficult to inhibit.

The "best-fit" sinusoid shown in Figure 1 for the baseline SC yielded predicted means that were highly correlated with the obtained means $(r=.91)$. The sinusoids shown for the other conditions were based on the control sinusoid, with their amplitudes shifted but not their phase or period, and similarly yielded predicted values that were close to the obtained values (for the prolonged noise background, $r=.93$, and for the brief inhibitory pulse, $r=$ $.85)$. That these correlations are high, however, does not mean unequivocally that the underlying function is sinusoidal. In fact, an imaginary set of data taken from a twovalued step function with the same overall mean as the obtained mean but with six identical high values in the dark and six identical low values in the light correlated significantly with the same sinusoid $(r=.80)$. This value is not significantly different from the obtained correlation coefficients $(p>.1)$. It is also noteworthy that the more serious discrepancies seen in Figure 1 between the obtained values and those predicted from the sinusoid tend to be toward the end of the dark or light cycle. This observation suggests that the current lighting condition was continuing to have some control over the response even as the significant response trend in the ANOVA was to anticipate the shift in lighting: Only this significant interaction of $\mathrm{H}$ and LD in the ANOVA supports the graphic indication of a sinusoidal rhythm in the data rather than a two-valued LD step function.

The data of the last 3 days were used in these analyses because it was assumed that the ASR amplitudes would be relatively stable during this time. This assumption proved to be correct. The baseline ASR declined by $51 \%$ from the 1st day of testing to the 1st day of the data set used in these analyses but then declined by only another $9 \%$ by the last test day. An ANOVA on changes in response strength over these last 3 days indicated that the modest decline in average ASR amplitudes over this period was significant for days $[F(2,6)=5.62, p<.05]$ but the various interactions with days were not significant $(F<1)$.

\section{DISCUSSION}

The present data confirm the prior reports (Chabot \& Taylor, 1992a, 1992b; Horlington, 1970) that the amplitude of the startle reaction of the rat is greater in the dark part of the daily illumination cycle than it is in the light. The ratio of the peak response obtained in the dark to the 
trough obtained in the light was about $2: 1$, which is comparable to the effect size seen in earlier work. This quantitative similarity in outcome is noteworthy because the various sets of data were obtained under very different conditions. In these two other sets of experiments, the animals were taken from the home environment at different times of the day and then given a brief test run, whereas in the present experiment they were maintained in the testing environment for a test period spanning several daily cycles. The similarity of these findings suggests that the brief testing intrusions used in the previous experiments must not have seriously interfered with the expression of the subject's normal diurnal cycle. The response probability data of Davis and Sollberger (1971), the only other experiment in which the testing period spanned several days, had more sharply defined peaks and troughs than the present amplitude data, but otherwise our temporal function is similar in theirs (provided in Sollberger \& Davis, 1972) in its fit to a sinusoid with a $24-\mathrm{h}$ period and zero-crossings near the LD transition. Because the ASR does not entirely reflect a simple two-valued light:dark step function but anticipates the next period by a reversal in the response trajectory midway through each condition, it appears the ASR is subject to an endogenous circadian rhythm that is independent of the current SC, but it should be noted that this small literature on startle rhythmicity does not contain the type of definitive study that examines target behavior across many cycles of uniform stimulation.

The primary purpose of the present experiment was to examine the influence of time of day on reflex modification, and the data pertinent to this question are most accessible in Figure 2. The upper panel of this figure shows that the absolute differences between the control baseline and either the inhibited response or the facilitated response (but here to a lesser extent) are greater in the dark than in the light: Thus, like many other behavioral and physiological functions in the rat (Rusek, 1981), reflex modification is affected by the diurnal cycle. Here, however, despite the graphic indication of a sinusoidal rhythm in the data, at least for reflex inhibition, there was no solid inferential support for this indication that the domain of the diurnal cycle extended beyond the effect of the current lighting condition.

A sometimes contentious issue in the analysis of reflex modification is whether reflex modification is best quantified by absolute response differences between control and modified values or by relative measures in which modification is expressed as a proportion of the control value. In the present experiment, for example, the answer to the simple empirical question, "Is reflex modification subject to a diurnal rhythm?" could be answered either by "yes" or "no" depending on which half of Figure 2 was being examined. We suggest that here, and perhaps in general, the apparently simple empirical question can be answered only in the context of a theoretical model that generates the observed relationships. A simple model appropriate for the present set of conditions assumes (1) that the brief stimulus subtracts some amount from the strength of the baseline ASR whereas the prolonged stimulus adds to that strength, a position advocated in Hoffman and Ison (1980); and (2) that these amounts are affected by the diurnal cycle, being greater in the dark than in the light. As a result, (3) the absolute deviations between control and inhibited or facilitated response values are greater in the dark than in the light, as is seen in the upper panel of Figure 2. In order to account for the "near miss" to a zero effect in the relative scores depicted in the lower panel of Figure 2, it is reasonable to assume further (4) that the extent to which the processes responsible for reflex modification are affected by the circadian rhythm is identical to its effect on the process responsible for reflex elicitation. Thus, for example, a doubling of the strength of the control response at a particularly favorable time of day compared with that at a less favorable time also doubles the effectiveness of the prepulse. Given this assumption, (5) the relative relationships between the control response and the modified responses must be invariant with time of day, save for the complications of potential floor and ceiling effects that lead to the appearance of compressive nonlinearities.

\section{REFERENCES}

Chabot, C. C., \& Taylor, D. H. (1992a). Circadian modulation of the rat acoustic startle response. Behavioral Neuroscience, 106, 846-852.

Chabot, C. C., \& TAYlor, D. H. (1992b). Daily rhythmicity of the rat acoustic startle response. Physiology \& Behavior, 51, 885-889.

Davis, M. (1974). Signal-to-noise ratio as a predictor of startle amplitude and habituation in the rat. Journal of Comparative \& Physiological Psychology, 86, 812-825.

Davis, M., \& Sollberger, A. (1971). Twenty-four hour periodicity of the startle response in rats. Psychonomic Science, 25, 37-39.

Gerrard, R. L., \& Ison, J. R. (1990). Spectral frequency and the modulation of the acoustic startle reflex by background noise. Journal of Experimental Psychology: Animal Behavior Processes, 16, 106-112.

Hoffman, H. S., \& Ison, J. R. (1980). Reflex modification in the domain of startle: I. Some empirical findings and their implications for how the nervous system processes sensory input. Psychological Review, 87, $175-189$

Hoffman, H. S., Marsh, R. R., \& Stein, N. (1969). Persistence of background acoustic stimulation in controlling startle. Journal of Comparative \& Physiological Psychology, 68, 280-283.

Hoffman, H. S., \& SEARLE, J. L. (1965). Acoustic variables in the modification of the startle reaction in the rat. Journal of Comparative \& Physiological Psychology, 60, 53-58.

Hoffman, H. S., \& Wible, B. L. (1970). Role of weak signals in acoustic startle. Journal of the Acoustical Society of America, 47, 489-497.

HoRLington, M. (1970). Startle response circadian rhythm in rats: Lack of correlation with motor activity. Physiology \& Behavior, 5, 49-53.

Ison, J. R., \& Hammond, G. R. (1971). Modification of the startle reflex in the rat by changes in the auditory and visual environments. Journal of Comparative \& Physiological Psychology, 75, 435-452.

Ison, J. R., McAdaM, D. W., \& Hammond, G. R. (1973). Latency and amplitude changes in the acoustic startle reaction produced by variation in auditory prestimulation. Physiology \& Behavior, 10, 1035-1039.

RuseK, B. (1981). Vertebrate behavioral rhythms. In J. Aschoff (Ed.), Handbook of behavioral neurobiology: Vol. 4. Biological rhythms (pp. 183-213). New York: Plenum.

Sollberger, A., \& Davis, M. (1972). Diurnal rhythm of the startle response: A statistical analysis. Journal of Interdisciplinary $C y c l e ~ R e-$ search, 3, 313-325.

(Manuscript received March 13, 1995; revision accepted for publication November 29, 1996.) 